\title{
Velocity Fluctuation of Hole in 1D Defect Turbulence
}

\author{
Yusuke Uchiyama* and Hidetoshi Konno \\ Dept. of Risk Engineering, Faculty of Information and Systems, \\ University of Tsukuba, Tsukuba, Ibaraki 305-8573, Japan \\ E-mail: * r1230160@risk.tsukuba.ac.jp
}

\begin{abstract}
We have definitely discriminated holes from defects in the defect turbulence described by the $1 \mathrm{D}$ complex Ginzburg-Landau equation. It is found that velocity of each hole is subjected to deterministic kinetics in our tracing on the path of a hole. On the other hand, probability density of the velocities in the whole system is identified by a generalized Cauchy distribution.
\end{abstract}

\section{Introduction}

The complex Ginzburg-Landau equation (CGLE) is the simplest model describing pattern dynamics in nonequilibrium open systems, especially near the Hopf bifurcation [1][2]. Various spatial and spatiotemporal patterns have been observed both numerically and experimentally [3][4]. In the systems, defect is a physical significance to understand the pattern dynamics: catalytic surface reaction [5], thermal fluid convection [6], inverse energy cascade in quantum turbulence [7] and so on. In particular, the Bekki-Nozaki (BN) hole solution, one of the exact solution of the 1D CGLE, is known to express a defect. Recently, it has been discovered in a human healthy heart [8]. This means that, from the point of view of nonlinear waves, the hole dynamics can give us helpful information to understand the nature of human hearts.

The 1D CGLE also displays several pattern dynamics involved in the BN-hole appearing as a phase singularity. Frozen state is a stationary state where steady configuration of the BN-holes and shocks are observed, which state emerges after transient dynamics dies out [9]. Spatiotemporal intermittency is modelled by a coupled map lattice composed of the BNholes and homoclinic holes in unstable background [10]. Defect turbulence or spatiotemporal chaos show disorder dynamics of both amplitude of phase, where local amplitudes reaching zero in producing phase singularities [11].

In spite of the observations related to the hole dynamics, statistical properties of those have not yet been clarified in detail because of difficulty in identifying the holes in the complicated dynamics. Actually, an identification method only with amplitude has caused unexpected illusions [12]. Neverthless, the traditional identification method has been employed in other works [11].
The counting number and the velocity fluctuations of defects have been investigated in inclined layer convections [13]. Probability density of the velocity fluctuations has been discussed in conjunction with BeckCohen superstatistics [14].

In this paper, we discriminate holes from defects definitely in the defect turbulence. Then the probability density of all the velocities of holes is identified by a generalized Cauchy distribution [15].

\section{BN-hole versus homoclinic hole}

The CGLE is derived from spatially extended dissipative systems by the reductive perturbation method. It describes both amplitude and phase modulations of the systems with respect to slowly varying space and time variables [16]. The scaled CGLE is expressed by a complex order parameter, $A(\vec{x}, t)$,

$$
\partial_{t} A=A+\left(1+i c_{1}\right) \nabla^{2} A-\left(1+i c_{2}\right)|A|^{2} A,
$$

with two real parameters, $\left(c_{1}, c_{2}\right)$, which are determined by intrinsic parameters of original physical systems.

In $1 \mathrm{D}$ systems, the CGLE has a defect solution traveling with constant velocity, the BN-hole [17, 18]:

$$
\begin{aligned}
A & =\frac{b_{1} \exp (\kappa \xi)+b_{2} \exp (-\kappa \xi)}{\exp (\kappa \xi)+\exp (-\kappa \xi)} \exp [i(q x-\Omega t)] \\
& \times \exp [-i \alpha \ln (2 \cosh (\kappa \xi))],
\end{aligned}
$$

where all the parameters in Eq. (2) are expressed by $c_{1}$ and $c_{2}$. This solution connects two plane waves with different wave numbers, and thus acts as a source of waves.

On the other hand, the homoclinic hole is classified into coherent structures which is expressed by the traveling wave solutions, $A(x, t)=\hat{A}(x-v t) \mathrm{e}^{-i \omega t}$, of Eq. (1) [10]. The ansatz provides three ordinary differential equations (ODEs):

$$
\begin{aligned}
& \partial_{\xi} a=\operatorname{Re}(z) a \\
& \partial_{\xi} z=-z^{2}+\frac{1}{1+i c_{1}}\left[-1-i \omega+\left(1+i c_{2}\right) a^{2}-v z\right],(4)
\end{aligned}
$$

where $\xi=x-v t, a=|\hat{A}|$ and $z \equiv \partial_{\xi} \ln (A)$. The homoclinic hole is an unstable homoclinic orbit of the above ODEs with respect to a saddle-node bifurcation. Since 

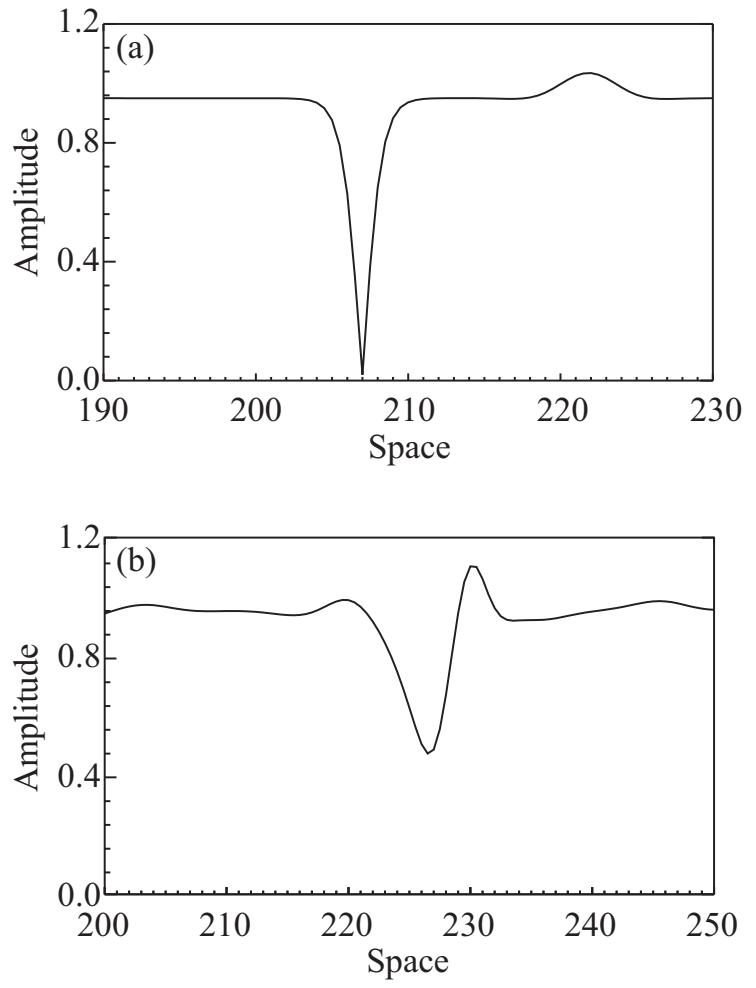

Fig. 1: Amplitude profiles of (a) the BN-hole and (b) the homoclinic hole. It is quite difficult to distinguish them since both shapes are characterized by localized amplitude dips.

there are no phase discontinuities, it is definitely discriminated from the BN-hole. However, amplitude profiles of the BN-hole and the homoclinic hole are quite similar.

\section{Numerical simulation}

We have implemented a numerical simulation of Eq. (1) by a split-step method [19]. The CGLE can be separated into two parts as

$$
\begin{aligned}
\partial_{t} A_{L} & =\left(1+i c_{1}\right) \nabla^{2} A_{L}, \\
\partial_{t} A_{N} & =A_{N}-\left(1+i c_{3}\right)\left|A_{N}\right|^{2} A_{N} .
\end{aligned}
$$

Both parts can be solved analytically and be expressed as $A_{L}(x, t+\Delta t)=\mathrm{e}^{\Delta t L} A_{L}(x, t)$ and $A_{N}(x, t+\Delta t)=$ $\mathrm{e}^{\Delta t N} A_{L}(x, t)$. The specific forms of the operators are shown in the form:

$$
\begin{aligned}
\mathrm{e}^{\Delta t L} & =\int_{\Omega_{y}} \mathrm{~d} y G(x-y ; \Delta t) \\
\mathrm{e}^{\Delta t N} & =\Phi(\Delta t) \exp \left[\Delta t+i c_{3} \ln \Phi(\Delta t)\right] \\
\Phi(\Delta t) & =\left[1-\left|A_{N}(x, t)\right|+\left|A_{N}(x, t)\right|^{2} \mathrm{e}^{2 \Delta t}\right]^{-1 / 2},
\end{aligned}
$$

where $G(x ; t)$ denotes the time-dependent Green's function in the system $\Omega_{x}$. Note that time evolution of Eq. (1) during $\Delta t$ is evaluated by $A(x, t+\Delta t)=$
$\mathrm{e}^{(L+N) \Delta t} A(x, t)$, where $\mathrm{e}^{\Delta t L}$ and $\mathrm{e}^{\Delta t N}$ do not need to be commutative. For sufficiently small $\Delta t$, $\mathrm{e}^{(L+N) \Delta t}$ is approximated in possessing fourth-order accurate on time by a split-step operator $S_{4}(\Delta t)$ :

$$
S_{4}(\Delta t)=S_{2}\left(\tau_{1} \Delta t\right) S_{2}\left(\tau_{0} \Delta t\right) S_{2}\left(\tau_{1} \Delta t\right)
$$

with

$$
S_{2}(\Delta t)=\mathrm{e}^{\frac{1}{2} \Delta t L} \mathrm{e}^{\Delta t N} \mathrm{e}^{\frac{1}{2} \Delta t L}
$$

and

$$
\tau_{1}=\frac{1}{2-2^{1 / 3}}, \quad \tau_{0}=1-2 \tau_{1} .
$$

The simulation has been performed under periodic boundary condition with time step $\Delta t=0.001$ and spatial resolution $\Delta x=0.061$. The parameters and the system size has been set, $\left(c_{1}, c_{2}\right)=(1.5,-1.2)$ and $L=80 \pi$, to generate a defect turbulence. Figure 2 shows a spatiotemporal pattern of (a) amplitude $|A|$ and (b) phase $\arg (A)$ with the corresponding snapshot of (c) amplitude and (d) phase at a certain time. Black and white lines in Fig. 2(a) correspond to local minima and maxima in Fig. 2(c), respectively. The black lines accompany discontinuities, namely phase jumps in Fig. 2(b). The range of phase value, as seen in Fig. 2(d), is extended to get unbounded lines for evaluating phase gradients adequately. We utilize the information about the local profiles of amplitude and phase to identify both the BN-holes and the homoclinic holes in detail.

The BN-hole is identified as a phase defect in disorder dynamics whereas the homoclinic hole does not have the characteristic [20]. Thus, in the defect turbulence, they are called defect and hole, respectively. Since the defects have a quite short lifetime, the black lines in Fig. 2(a) correspond to trajectories of the holes. Velocities of the holes, gradients of the black lines in Fig. 2(a), are seen not to be constant values. The velocities therefore seem to fluctuate in the defect turbulence.

\section{Velocity Kinetics}

The velocities of the holes at each time step are evaluated from local slopes of trajectories of the holes. Figure 3 shows three representative paths of the holes with the corresponding velocity diagrams. One can see different kinetics: a moving hole with nearly constant velocity in Fig. 3(a) and (d); a hole decreasing velocity monotonously in Fig. 3(b) and (e); a hole changing the direction of acceleration in Fig. 3(c) and (f). In the context of the coherent structures, the velocities of the holes are constant, however, they display complex kinetics in the defect turbulence. It is thus necessary to expand the concept of the coherent structures with constant velocity to that with time-dependent velocity in order to describe real traveling holes in the defect turbulence. In real worlds, single hole with constant velocity is rare to be discovered because it travels in disordered background generated from motions with many degree of freedom. In other words, velocity changes by local 

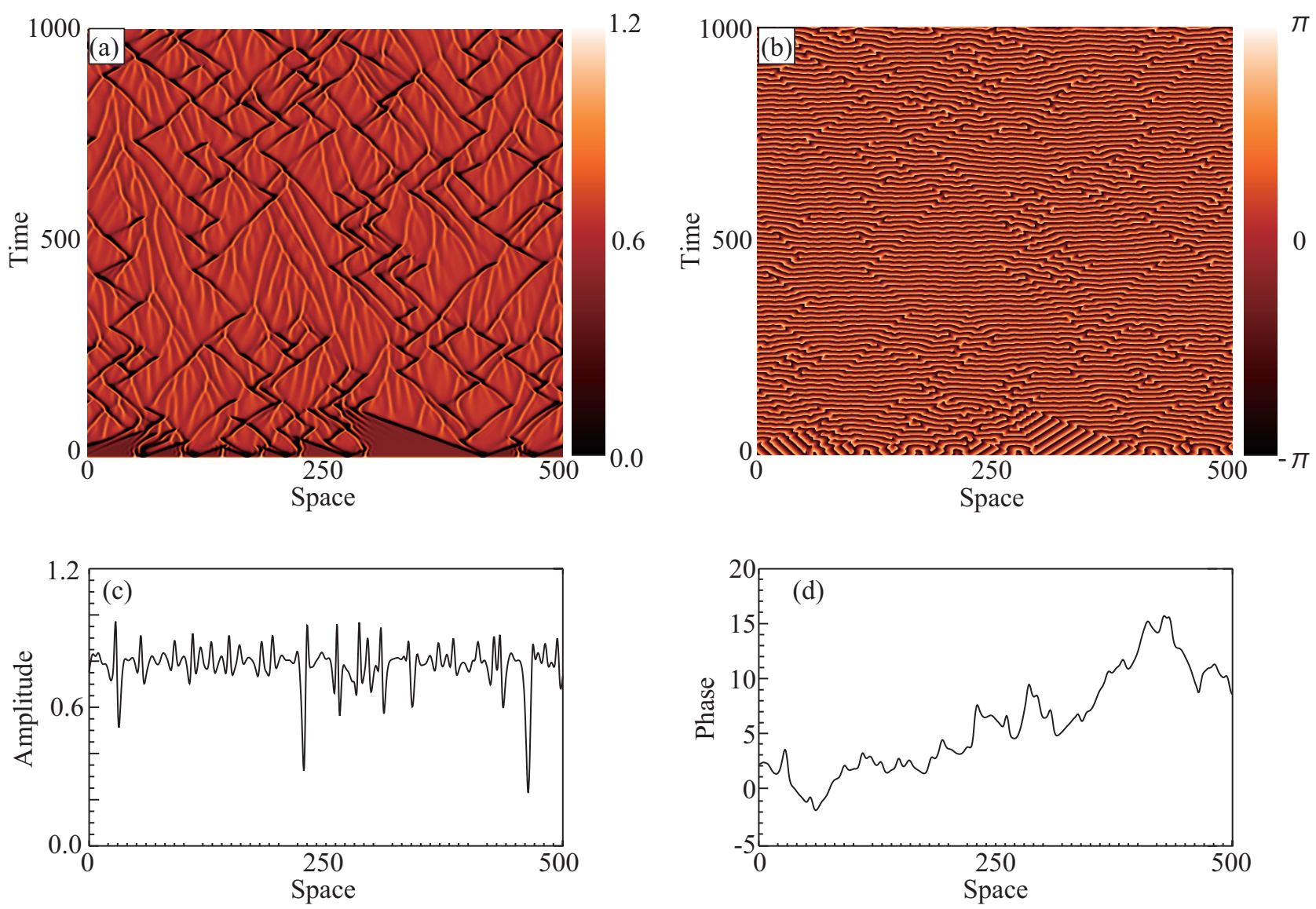

Fig. 2: A spatiotemporal pattern of (a) amplitude $|A|$ and (b) phase $\arg (A)$ with the corresponding snapshot of (c) amplitude and (d) phase at certain time. Black and white lines in (a) correspond to the local minima and maxima of amplitude in (c), respectively. The discontinuities of color gradation in (b) and the phase jumps in (d) are accompanied with the local minima of amplitude. 


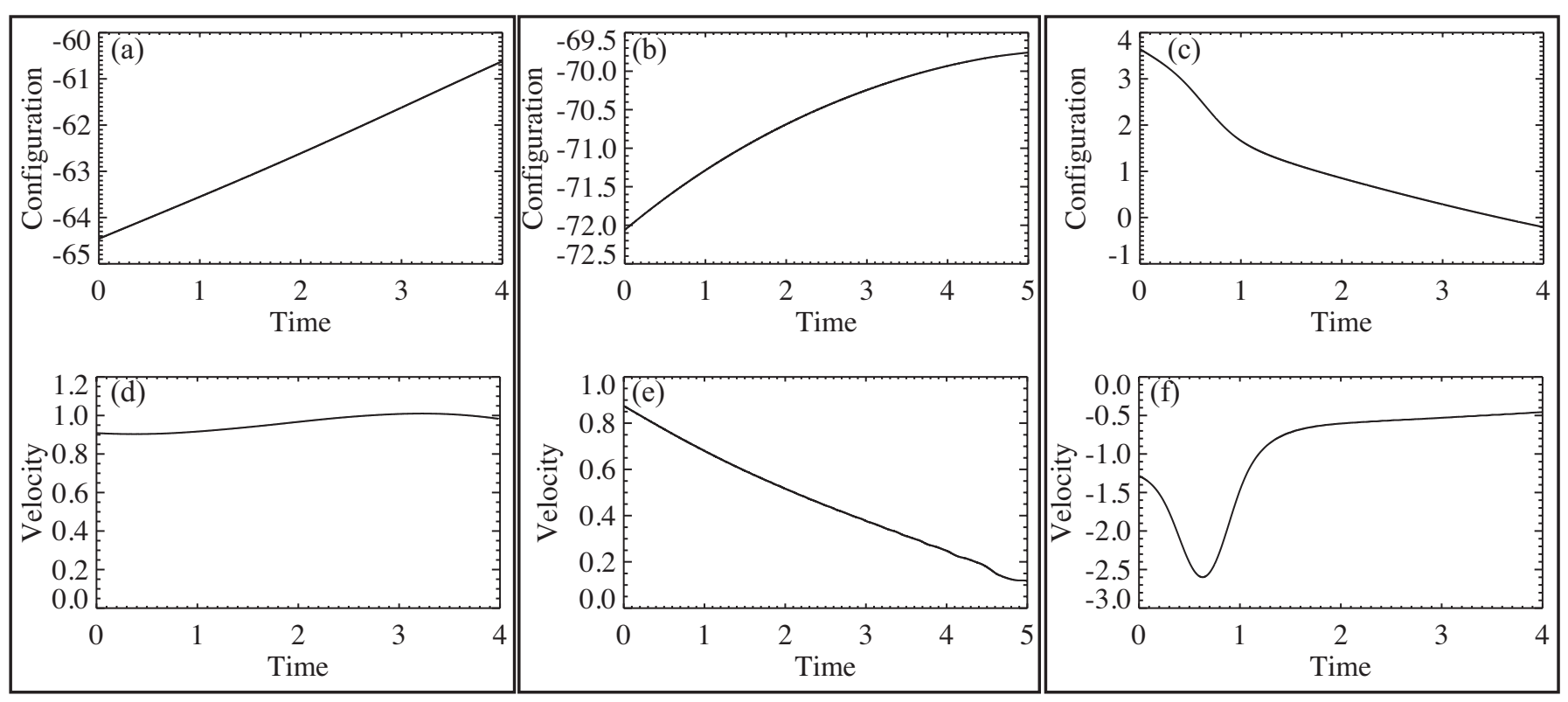

Fig. 3: Three representative paths of the holes with the corresponding velocity diagrams. Left column shows (a) a path and (d) a velocity diagram of a moving hole with nearly constant velocity. Central column shows (b) a path and (e) a velocity diagram of a hole decreasing velocity monotonously. Right column shows (c) a path and (f) a velocity diagram of a hole changing the direction of acceleration.

interactions between nonlinear waves. Thus, statistical analysis can be useful to understand the velocity kinetics in the whole space related to real situations.

The tracing procedure of the holesbased on the Lagrangian description shows that the velocity kinetics are deterministic. On the other hand, without distinguishing each hole, based on the Eulerian description, values of the velocities in the whole system are expected to have an universal statistical law. Actually, a probability density of the velocities in the whole space is identified by a generalized Cauchy distribution [15]:

$$
P_{s}(v)=\frac{a^{2 b-1}}{B(b-1 / 2,1 / 2)} \frac{1}{\left(a^{2}+v^{2}\right)^{b}},
$$

with the Beta function $B(x, y)$ and two parameters $a$ and $b$. Figure 4 compares the probability density obtained from the numerical simulation with the theoretical one. Fat-tail profile in Fig. 4, large deviations from the Gaussian statistics, is ascribed to the existence of inhomogeneity of the velocity kinetics in Fig. 3.

There are several stochastic processes which give the generalized Cauchy distribution at the steady state. It is thus necessary to identify the corresponding stochastic process based on the Eulerian description. This stochastic modelling for this picture will be investigated in the future.

\section{Conclusion}

We have investigated the defect turbulence described by the 1D CGLE. The defects and the holes have been

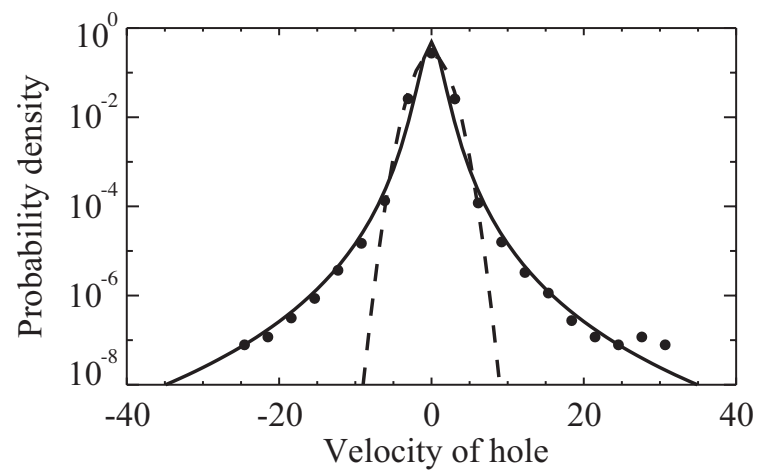

Fig. 4: The probability density of the velocities of the holes from the numerical simulation (black circles) and the generalized Cauchy distribution (solid line) with the estimated parameters, $(a, b)=(1.73,2.93)$. The profile of the Gaussian distribution is also shown in dashed line. 
definitely discriminated. Then, it is shown that the velocities of the holes obey the deterministic kinetics based on the Lagrangian description. On the other hand, based on the Eulerian description, the probability density of the velocities of the holes in the whole system is found to have the generalized Cauchy statistics. This is due to the inhomogeneous nature of the velocity kinetics in the 1D CGLE.

\section{Acknowledgements}

Y. U. is grateful to Grant-in-Aid for JSPS Fellows No. 25.374. H. K. is grateful to Grant-in-Aid for Challenging Exploratory Research No. 24650147.

\section{References}

[1] M. C. Cross and P. C. Hohenberg: Pattern formation outside of equilibrium, Rev. Mod. Phys., 65, pp.851-1112, 1993.

[2] M. Cross and H. Greenside: Pattern Formation and Dynamics in Nonequilibrium Systems, Cambridge University Press, 2009.

[3] I. S. Aranson and L. Kramer: The world of the complex Ginzburg-Landau equation, Rev. Mod. Phys., 74, pp.99-143, 2002.

[4] F. Sagués, J. M. Sancho and J. García-Ojalvo: Spatiotemporal order out of noise, Rev. Mod. Phys., 74, pp.829-882, 2007.

[5] C. Beta, A. S. Mikhailov, H. H. Rotermund and G. Ertl: Defect-mediated turbulence in a catalyitic surface reaction, Europhys. Lett., 75, pp.868-874, 2006.

[6] C. Huepe and H. Riecke: Statistics of defect trajectories in spatio-temporal chaos in inclined layer convection and the complex Ginzburg-Landau eqation, Chaos, 14, pp.864-874, 2004.

[7] M. T. Reeves, T. P. Billam, B. P. Anderson and A. S. Bradley: Inverse energy cascade in forced two-dimensional quantum turbulence, Phys. Rev. Lett., 110, 104501, 2013.

[8] N. Bekki, Y. Harada and H. Kanai: Bekki-Nozaki hole in traveling excited waves on human cardiac interventricular septum, J. Phys. Soc. Jpn., 81, 073801, 2012.

[9] H. Chaté: Spatiotemporal intermittency regimes of the one-dimensional complex Ginzburg-Landau equation, Nonlinearity, 7, pp.185-204, 1994.

[10] M. van Hecke: Building blocks of spatiotemporal intermittency, Phys. Rev. Lett., 80, pp.1896-1899, 1998.
[11] J. A. Sherratt and M. J. Smith: Transition to spatiotemporal chaos via stationary branching shocks and holes, Physica D, 241, pp.1671-1679, 2012.

[12] Y. Uchiyama and H. Konno: Statistical description of wave interactions in 1D defect turbulence, Proc. World Acad. Sci. Eng. Tech., 80, pp.207-212, Amsterdam, 2013.

[13] K. E. Daniels, C. Beck and E. Bodenschatz: Defect turbulence and generalized statistical mechanics, Physica D, 193, pp.208-217, 2004.

[14] C. Beck: Generalized statistical mechanics for superstatistical systems, Phil. Trans. R. Soc. A, 369, pp.453-465, 2011

[15] H. Konno and F. Watanabe: Maximum likelihood estimators for generalized Cauchy processes, $J$. Math. Phys., 48, 103303, 2007.

[16] Y. Kuramoto: Chemical Oscillations, Waves, and Turbulence, Dover, 2003.

[17] K. Nozaki and N. Bekki: Exact solutions of the generalized Ginzburg-Landau equation, J. Phys. Soc. Jpn., 53, pp.1581-1582, 1984.

[18] J. Lega: Traveling hole solution of the complex Ginzburg-Landau equation: a review, Physica D, 152-153, pp.269-287, 2001.

[19] J. Yang: Nonlinear Waves in integrable and Nonintegrable Systems, SIAM, 2010.

[20] M. van Hecke: Coherent and incoherent structures in systems described by the 1D CGLE: experiments and identification, Physica D, 174, pp.134$151,2003$. 